\title{
Assessment of sulfur poisoning of Ni/CGO-based SOFC anodes
}

Matthias Riegraf, Vitaliy Yurkiv, Rémi Costa, Günter Schiller, K. Andreas Friedrich

German Aerospace Center (DLR), Stuttgart, Germany
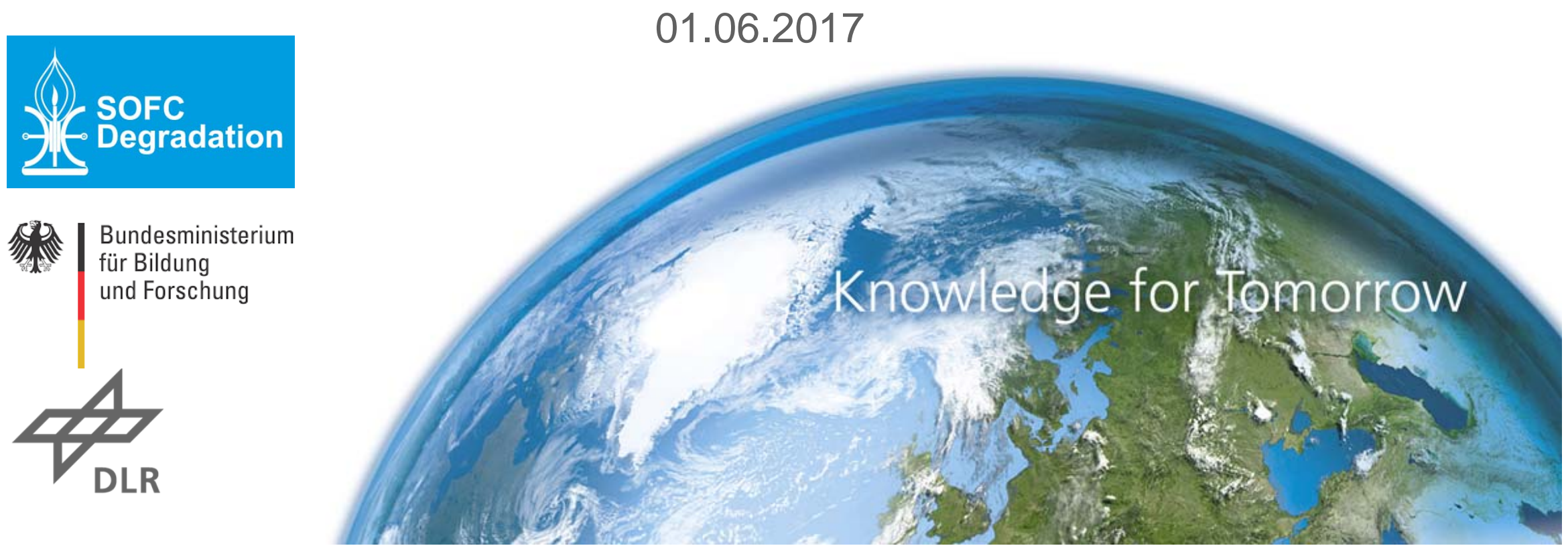


\section{Motivation and aim of the work}

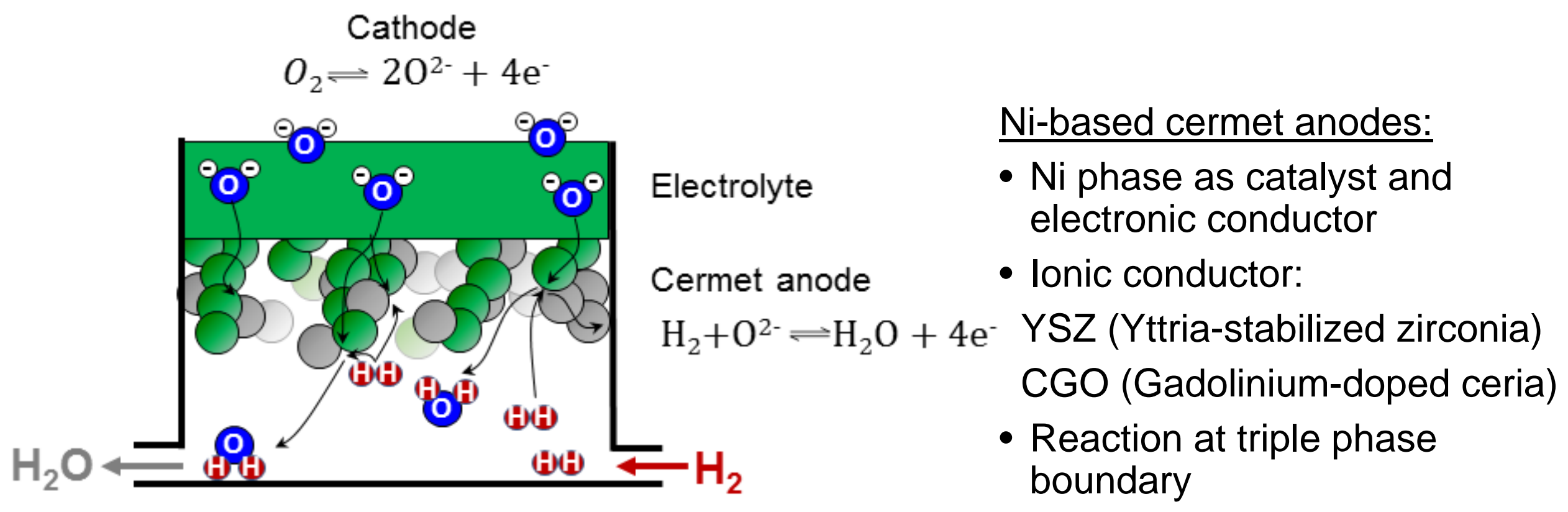

- Sulfur-containing impurities (mainly $\mathrm{H}_{2} \mathrm{~S}$ ) in natural gas and biogas $\rightarrow \mathrm{Ni}$ surface poisoning

- Higher sulfur tolerance of Ni/CGO than Ni/YSZ towards in $\mathrm{H}_{2}$ fuels

Goal: - Investigation of sulfur poisoning in reformate fuels

- Derivation of a reaction mechanism

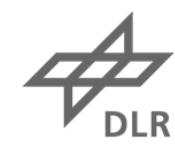




\section{Methodology}

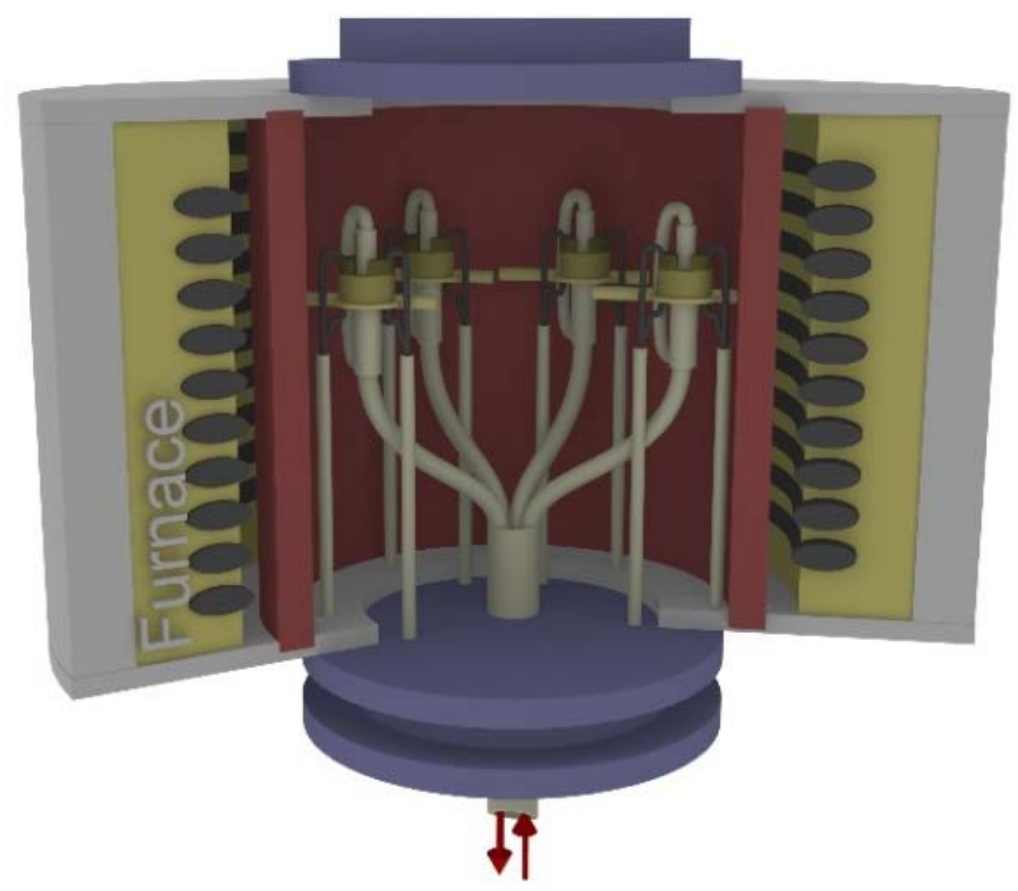

- Simultaneous measurement of up to four cells under variation of $p \mathrm{H}_{2} \mathrm{~S}$ and current density $i$

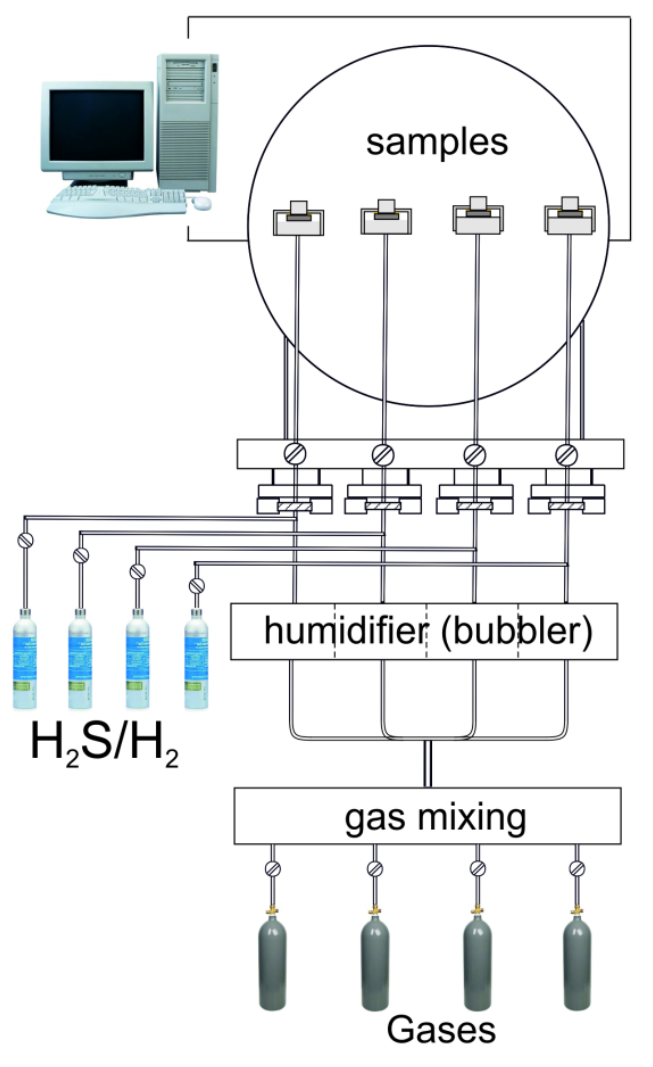

$\rightarrow$ Comprehensive parameter studies possible
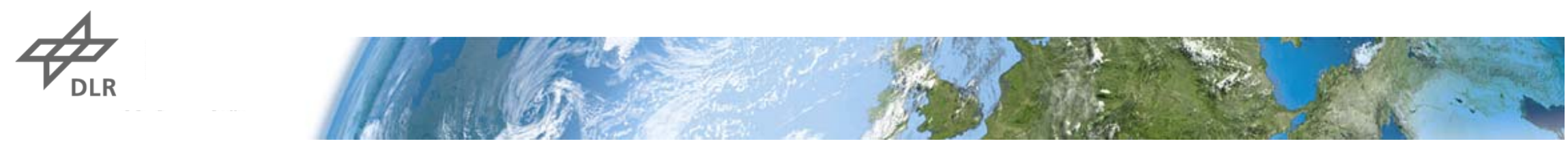


\section{Methodology}

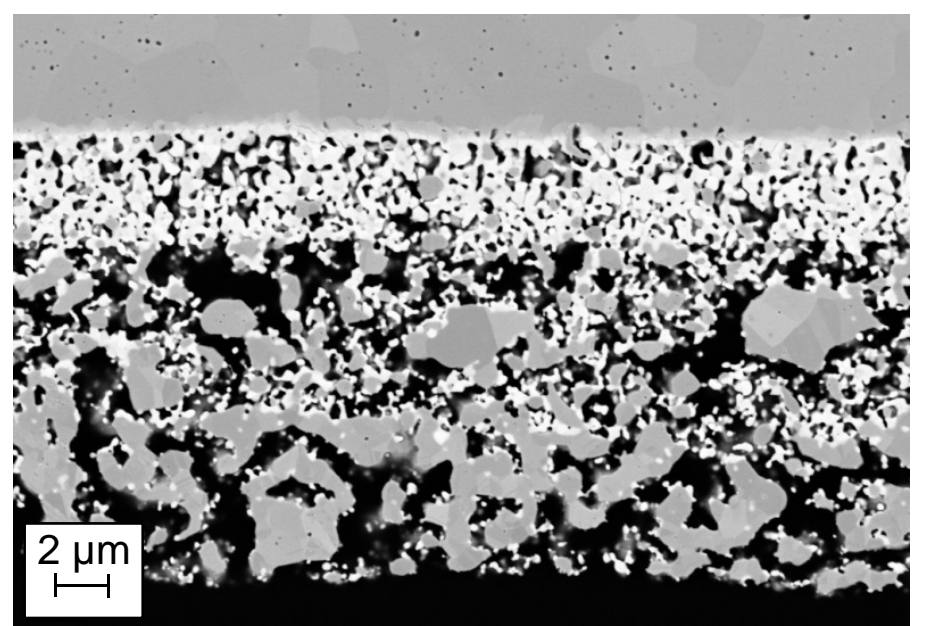

Ni/CGO10 anode $(20 \mu \mathrm{m})$

- Fraunhofer IKTS, Germany

- $160 \mu \mathrm{m}$ 10Sc1CeSZ electrolyte

- $65 \mu \mathrm{m}$ LSMM' cathode

- 5 um CGO10 barrier layer

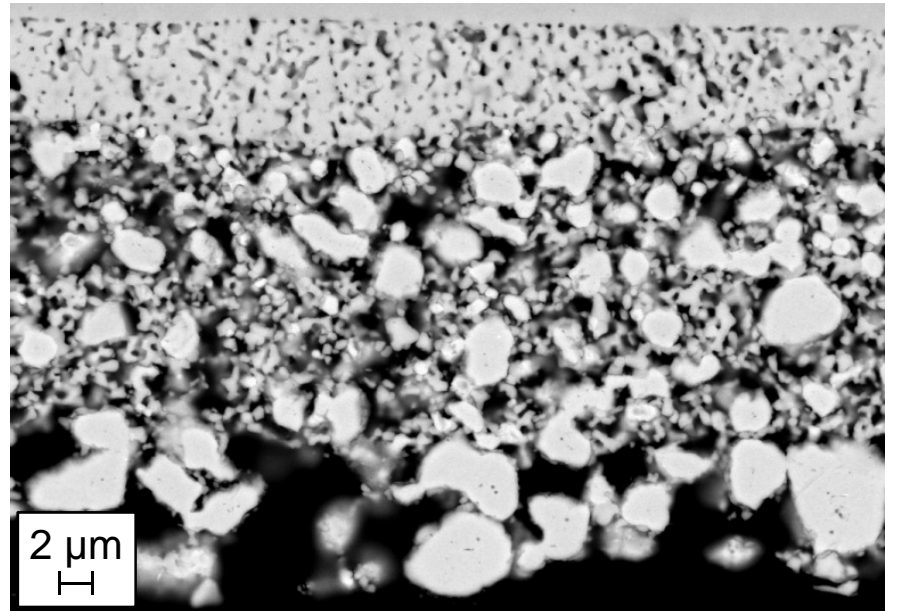

Ni/8YSZ anode $(25 \mu \mathrm{m})$

- Kerafol, Germany

- $160 \mu \mathrm{m}$ 10Sc1CeSZ electrolyte

- $65 \mu \mathrm{m}$ LSM/10Sc1CeSZ cathode

- $5 \mu \mathrm{m}$ 8YSZ barrier layer 


\section{Sulfur poisoning in $\mathrm{H}_{2} / \mathrm{H}_{2} \mathrm{O}$ fuels: Ni/CGO vs. Ni/YSZ}

$850{ }^{\circ} \mathrm{C}, 0.5 \mathrm{~A} \cdot \mathrm{cm}^{-2}, 97 \% \mathrm{H}_{2}, 3 \% \mathrm{H}_{2} \mathrm{O},+1,2,5,10,20 \mathrm{ppm} \mathrm{H}_{2} \mathrm{~S}$
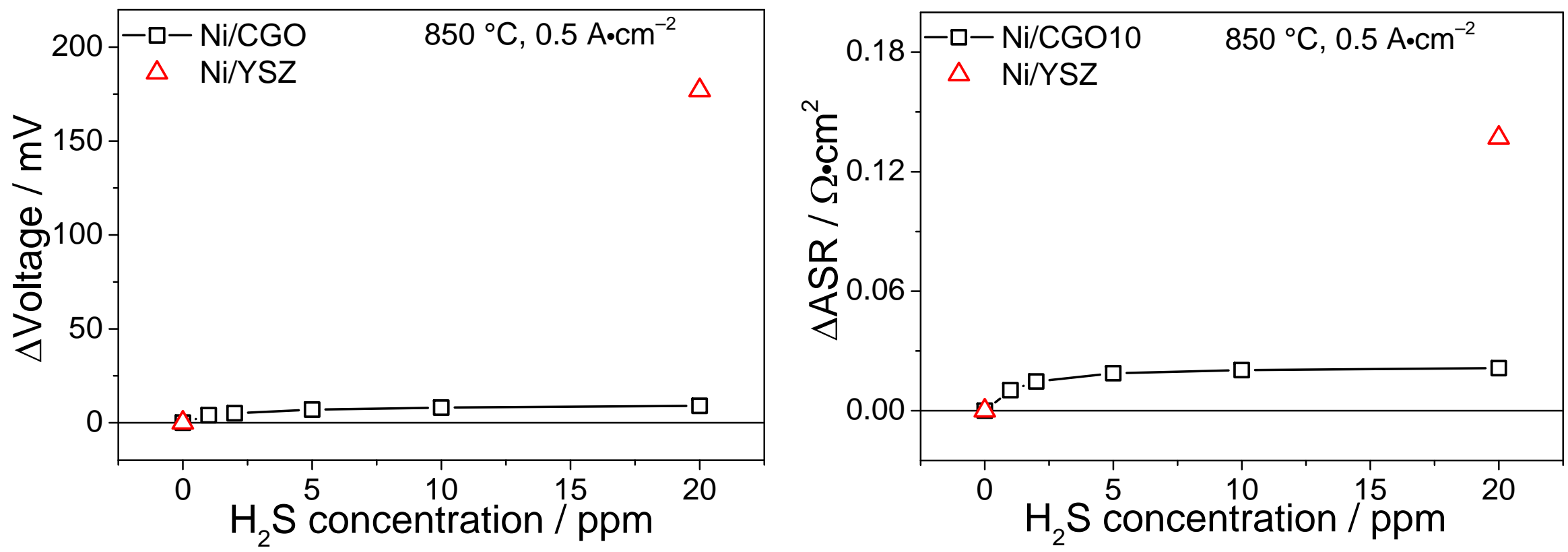

- Despite same Ni content, significantly larger resistance increase for $\mathrm{Ni} / \mathrm{CGO}$ anodes! $\rightarrow$ Reaction mechanisms mu

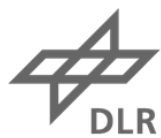




\section{Mechanism of Ni/CGO}

\section{Literature theories for high sulfur tolerance:}

1. Sulfur oxidation to $\mathrm{SO}_{2}$ via oxygen spillover from CGO to $\mathrm{Ni}[1,2]$

2. Sulfur diffusion into CGO bulk phase $[3,4]\}$ Free Ni surface

3. Catalytic activity of CGO towards $\mathrm{H}_{2}$ oxidation $[5,6]$ 


\section{Reformate operation Ni/CGO}

- $T=850^{\circ} \mathrm{C}, i=0.25,0.5,0.75 \mathrm{~A} \cdot \mathrm{cm}^{-2}$

- $16 \% \mathrm{H}_{2}, 16 \% \mathrm{CO}, 14 \% \mathrm{CO}_{2}, 14 \% \mathrm{H}_{2} \mathrm{O}, 40 \% \mathrm{~N}_{2}$

- Model diesel reformate for APU application

- Large CO content facilitates investigation of CO conversion

Water gas shift reaction: $\quad \mathrm{CO}+\mathrm{H}_{2} \mathrm{O} \rightleftharpoons \mathrm{H}_{2}+\mathrm{CO}_{2}$

CO oxidation: $\quad \mathrm{CO}+\mathrm{O}^{2-} \rightleftharpoons \mathrm{CO}_{2}+2 \mathrm{e}^{-}$ 


\section{Reformate operation Ni/CGO}
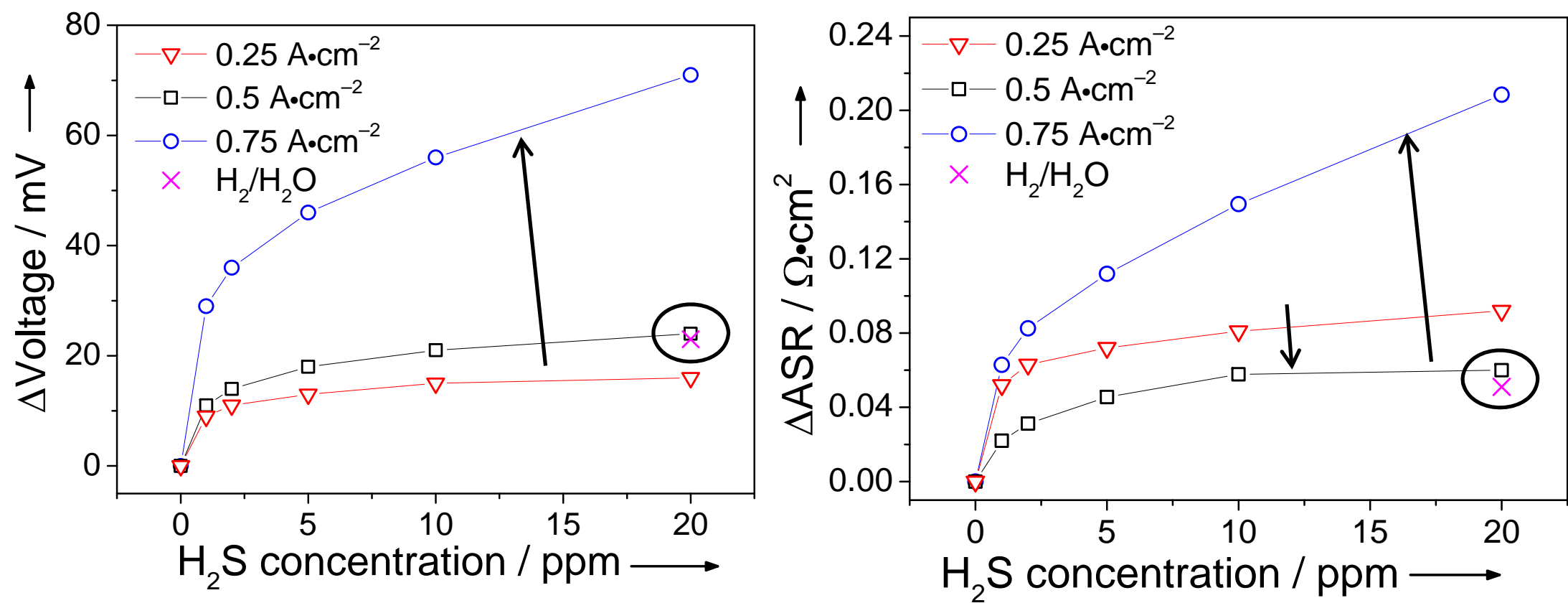

- $16 \% \mathrm{H}_{2}, 16 \% \mathrm{CO}, 14 \% \mathrm{CO}_{2}, 40 \% \mathrm{~N}_{2}, 14 \% \mathrm{H}_{2} \mathrm{O}$

$32 \% \mathrm{H}_{2}$

$70 \% \mathrm{~N}_{2}$

- Comparison with $\mathrm{H}_{2} / \mathrm{H}_{2} \mathrm{O}$ fuel at $0.5 \mathrm{~A} \cdot \mathrm{cm}^{-2} \rightarrow \mathrm{H}_{2}$ content defines performance drop

- More severe effect of sulfur poisoning at higher current densities (=higher fuel utilization) $\rightarrow$ inhibited CO conversion 


\section{Reformate operation Ni/YSZ}

- $7 \% \mathrm{H}_{2}, 7 \% \mathrm{H}_{2} \mathrm{O}, 86 \% \mathrm{~N}_{2}$

- $7 \% \mathrm{H}_{2}, 7 \% \mathrm{H}_{2} \mathrm{O}, 20 \% \mathrm{CO}_{2}, 20 \% \mathrm{CO}$

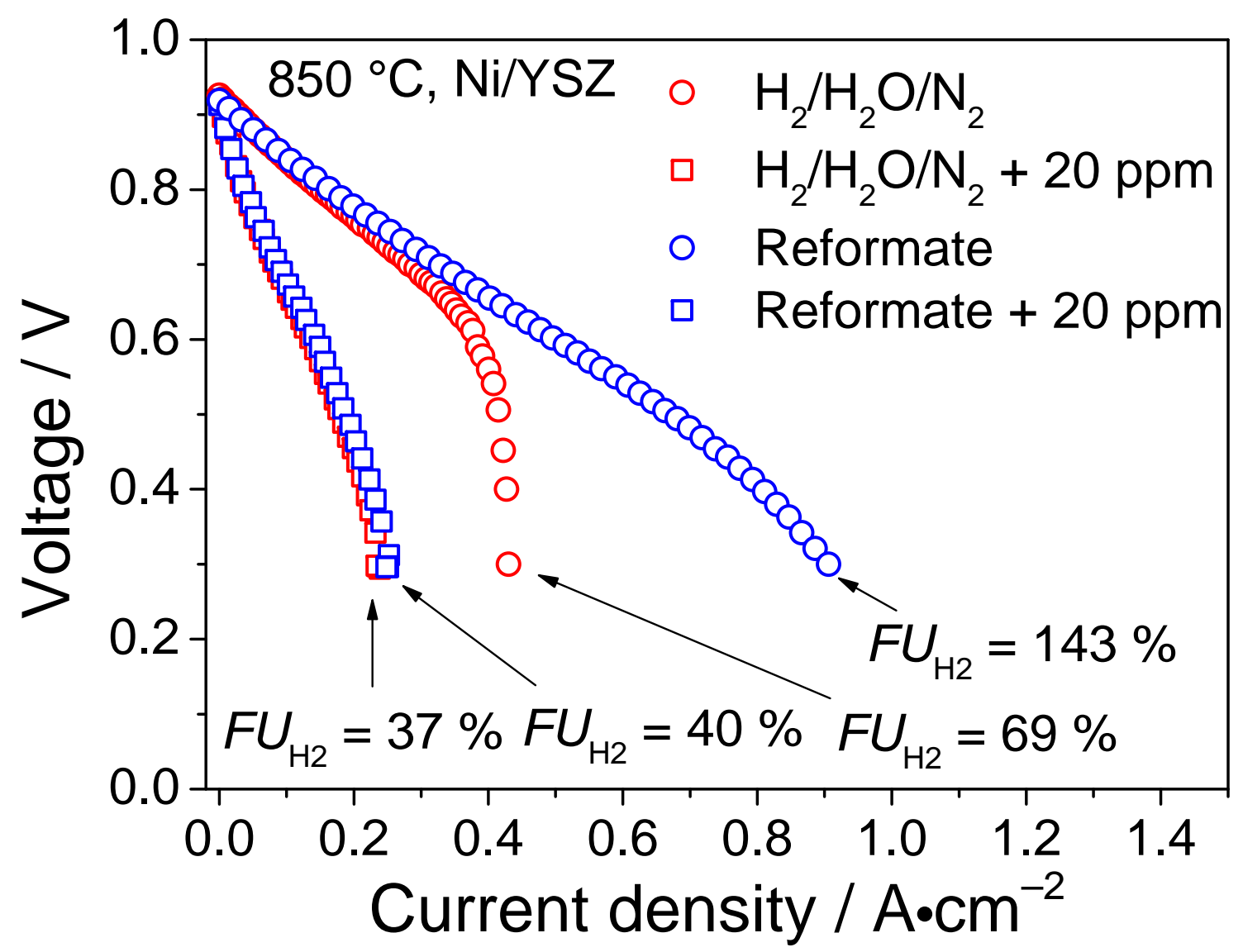

20 ppm:

Same performance in reformate and nonreformate

$\rightarrow$ Water gas shift reaction and $\mathrm{CO}$ oxidation are completely blocked 


\section{Reformate operation Ni/CGO}

- $7 \% \mathrm{H}_{2}, 7 \% \mathrm{H}_{2} \mathrm{O}, 86 \% \mathrm{~N}_{2}$

- $7 \% \mathrm{H}_{2}, 7 \% \mathrm{H}_{2} \mathrm{O}, 20 \% \mathrm{CO}_{2}, 20 \% \mathrm{CO}$

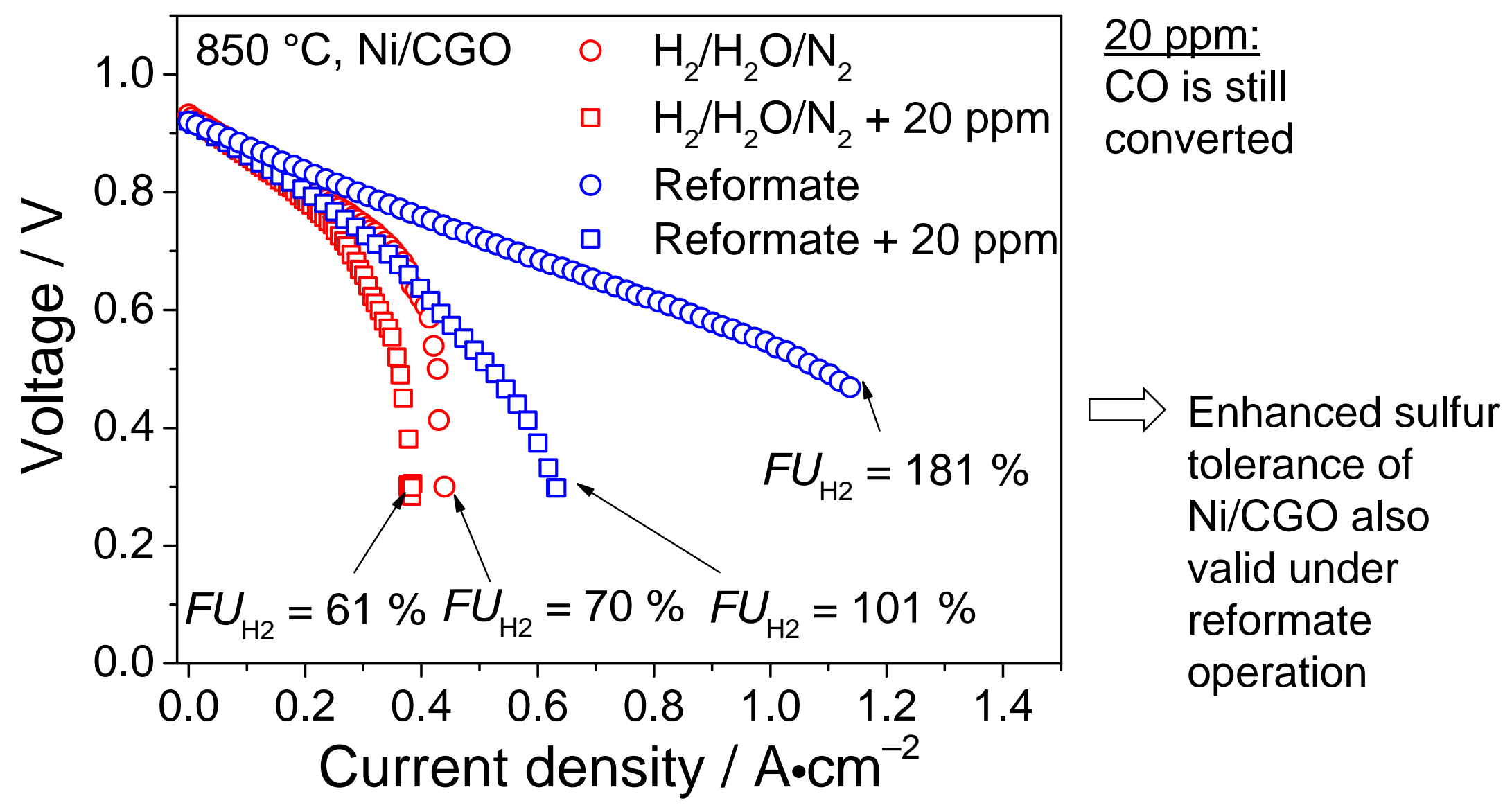




\section{CO oxidation}

$\mathrm{Ni} / \mathrm{CGO}$

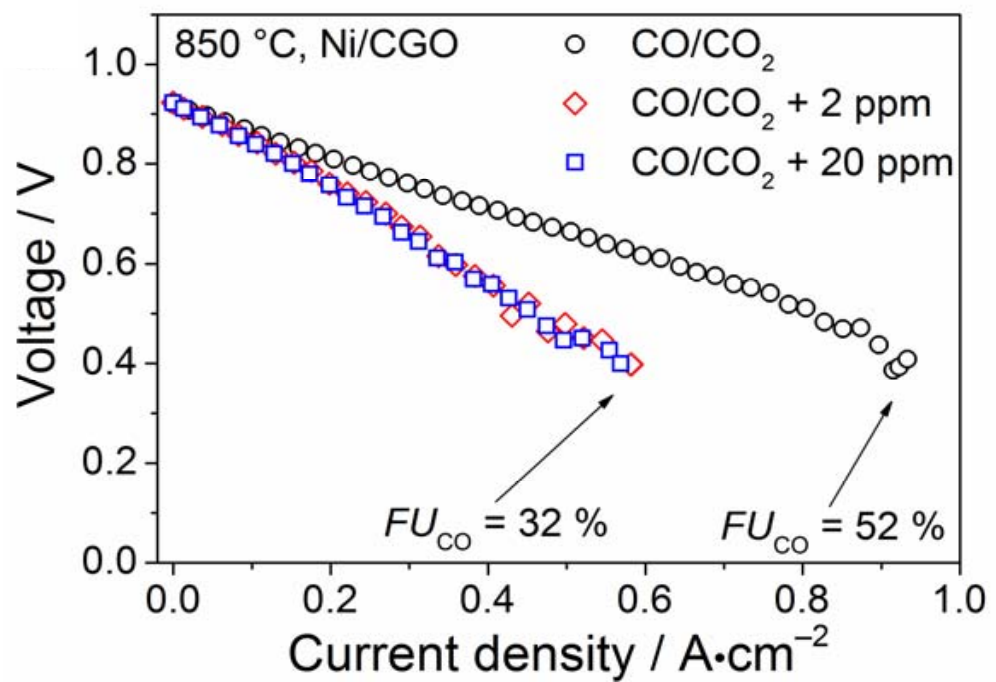

$\mathrm{Ni} / \mathrm{YSZ}$

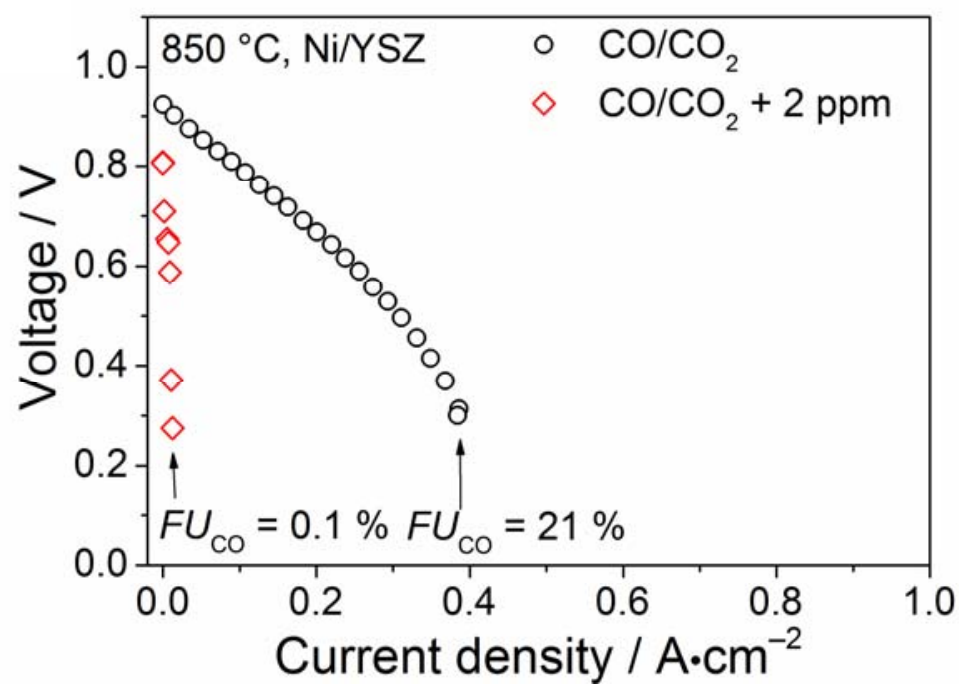

- Ni/YSZ: electrochemical CO oxidation fully blocked

- Ni/CGO: electrocemical CO oxidation poisoned, but still active

$\rightarrow$ Water gas shift deactivation, electrochemical CO oxidation on CGO
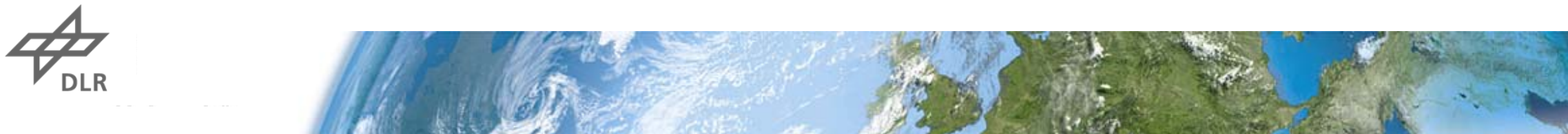


\section{CO oxidation}

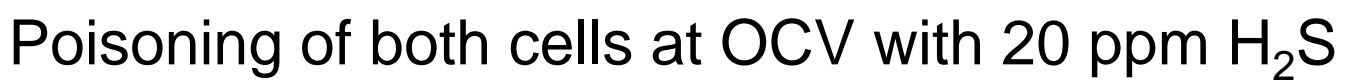

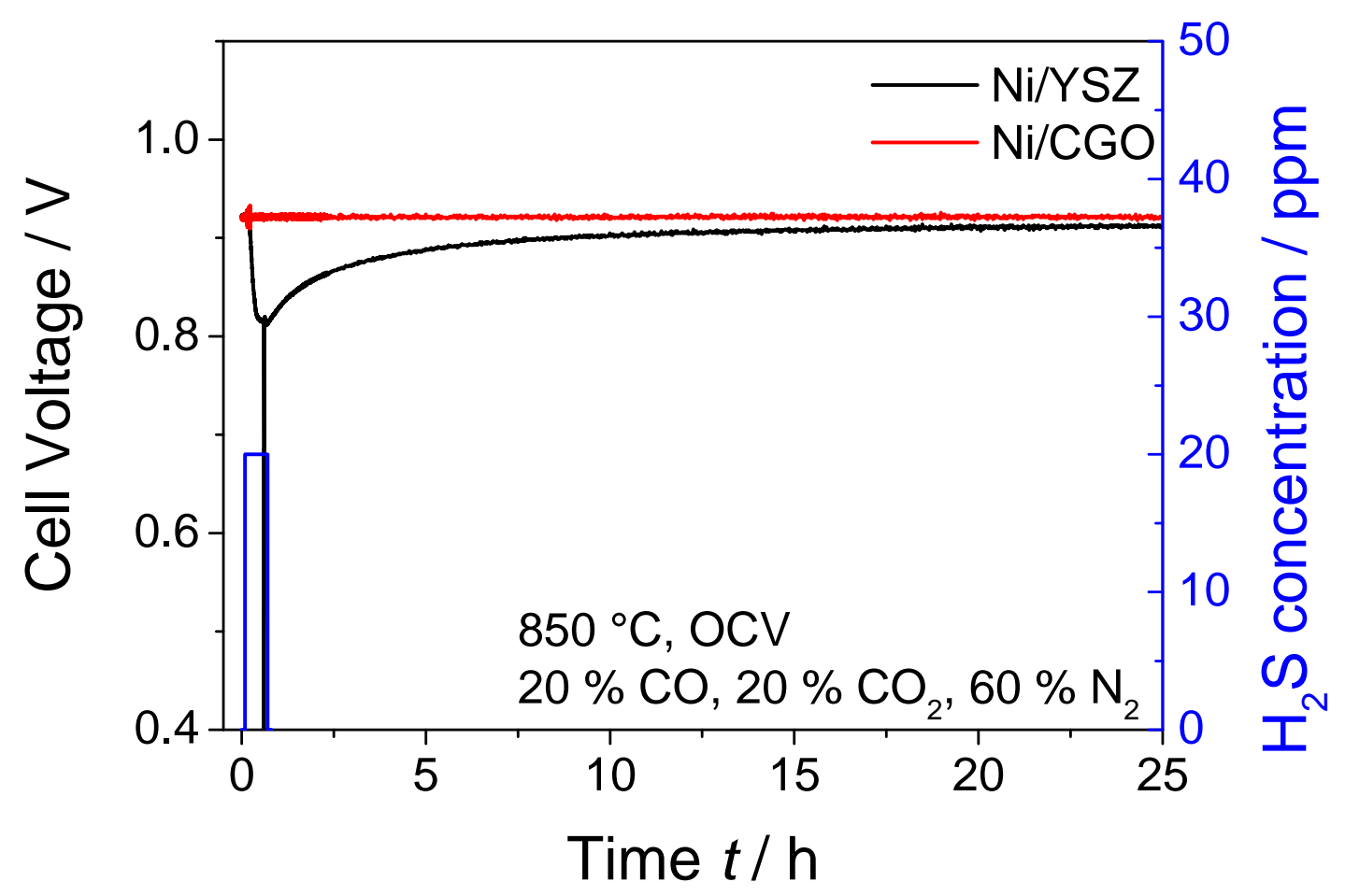

- OCV drop for Ni/YSZ

- CO oxidation is fully deactivated due to Ni surface poisoning

$$
\mathrm{COS}_{\mathrm{Ni}}+3 \mathrm{O}^{2-} \rightarrow \mathrm{CO}_{2, \mathrm{~g}}+\mathrm{SO}_{2, \mathrm{~g}}+6 \mathrm{e}^{-}
$$




\section{Methane steam reforming}

Poisoning of Ni/CGO at OCV with 1-20 ppm $\mathrm{H}_{2} \mathrm{~S}$

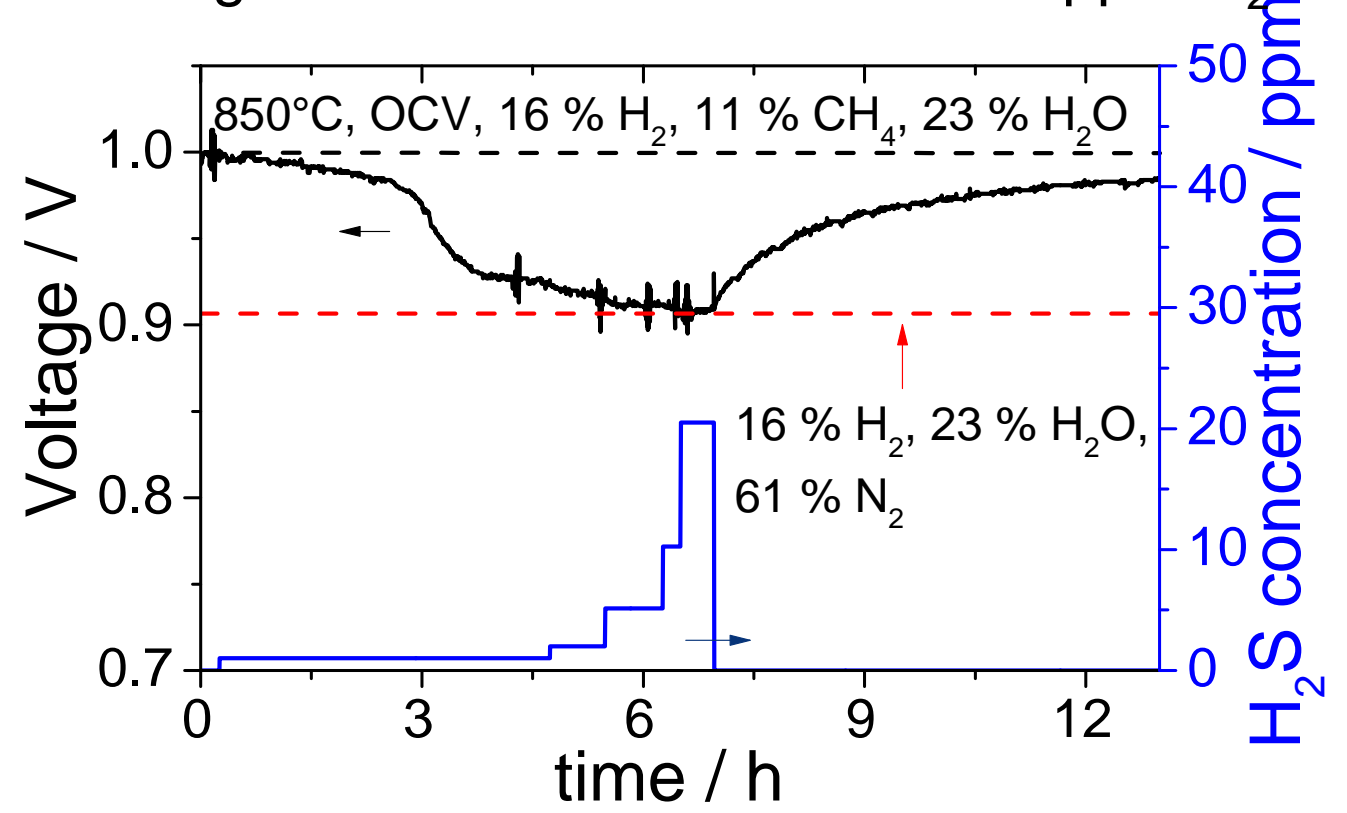

- Complete poisoning of Ni surface also on Ni/CGO

- Similar poisoning behavior of WGS and methane steam reforming on $\mathrm{Ni} / \mathrm{YSZ}[1,2]$

$\rightarrow$ Ni surface also fully deactivated for CO adsorption on Ni/CGO 


\section{Mechanism of Ni/CGO}

\section{Literature theories for high sulfur tolerance:}

Deactivation Methane

1. Sulfur oxidation to $\mathrm{SO}_{2}$ via oxygen spillover from CGO to Ni $[1,2]$

2. Sulfur diffusion into CGO bulk phase $[3,4]$

3. Catalytic activity of CGO towards $\mathrm{H}_{2}$ oxidation $[5,6]$

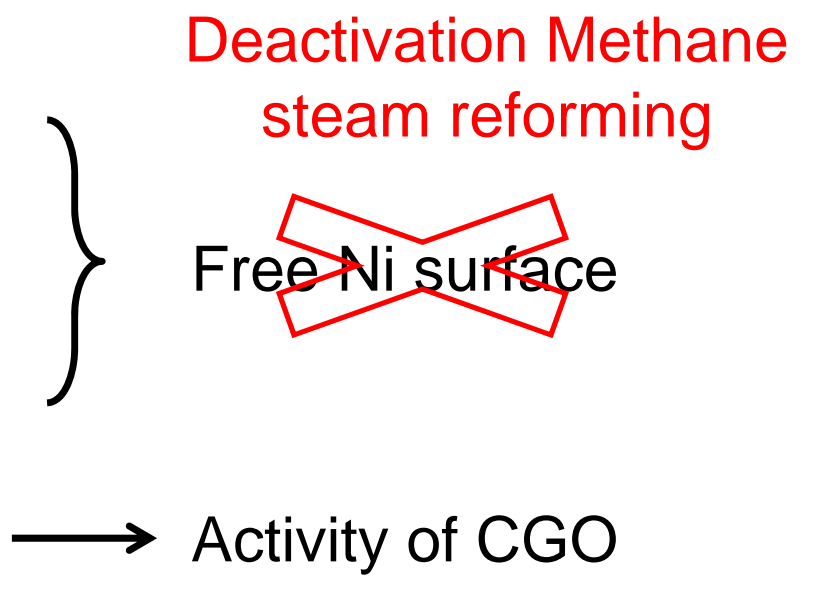

[1] Kavarucu et al., J. Power Sources, 217, (2012), 364; [2] Xu et al., J. Electrochem. Soc., 158, (2011); [3] Gerstl et al., Materials, 9, (2016); [4] Mullins et al., Surf. Science, 601, (2016); [5] Chueh et al., Nat. Materials, 11, (2011); [6] Nakamura et al., J. Electrochem. Soc, 1555, (2008) 


\section{CO oxidation mechanism on Ni/CGO}

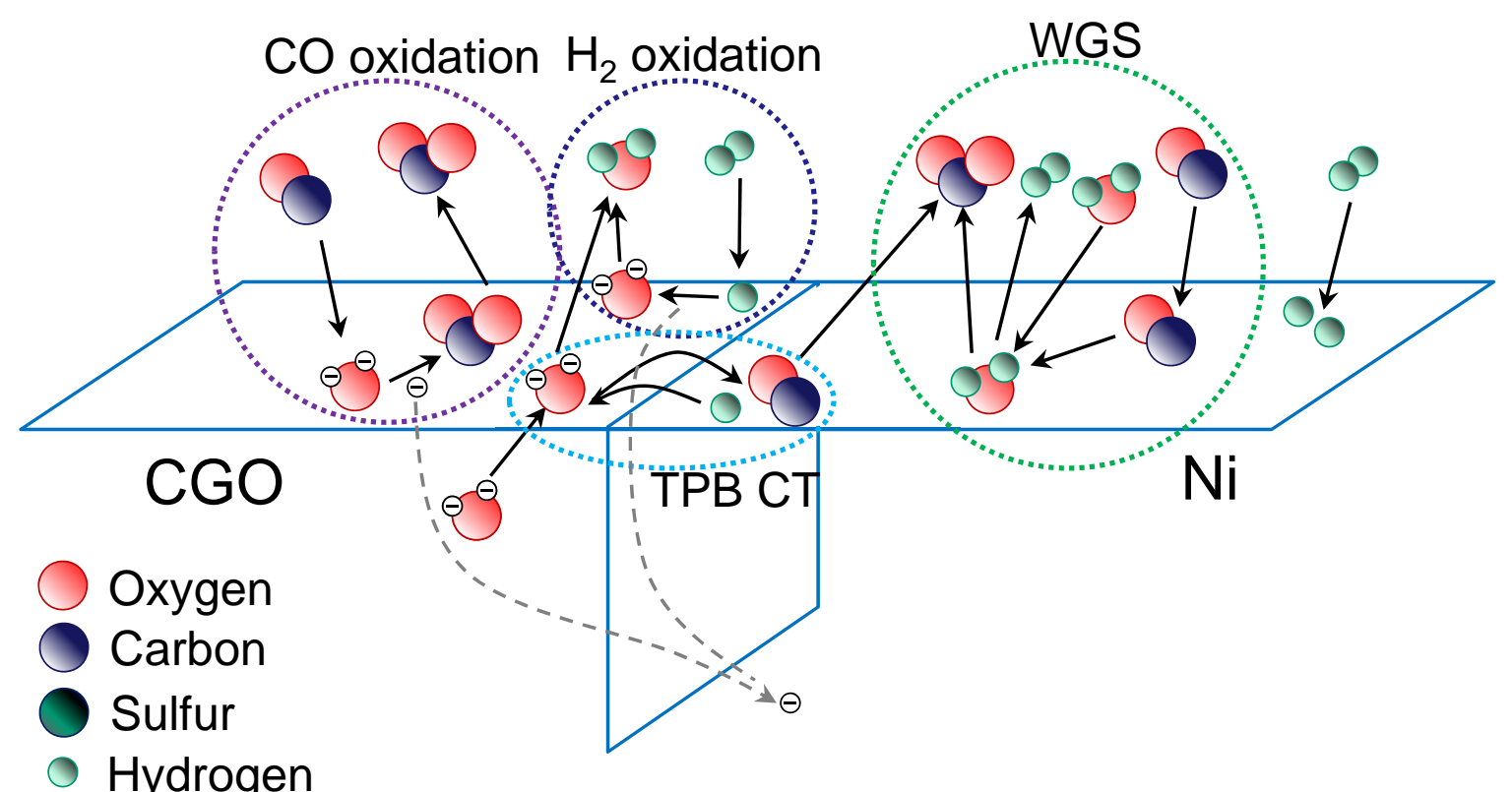

- CO oxidation on CGO surface possible

- But CO oxidation at TPB dominates, as CO strongly adsorbs on $\mathrm{Ni}$ 


\section{CO oxidation mechanism on Ni/CGO: Sulfur poisoning}

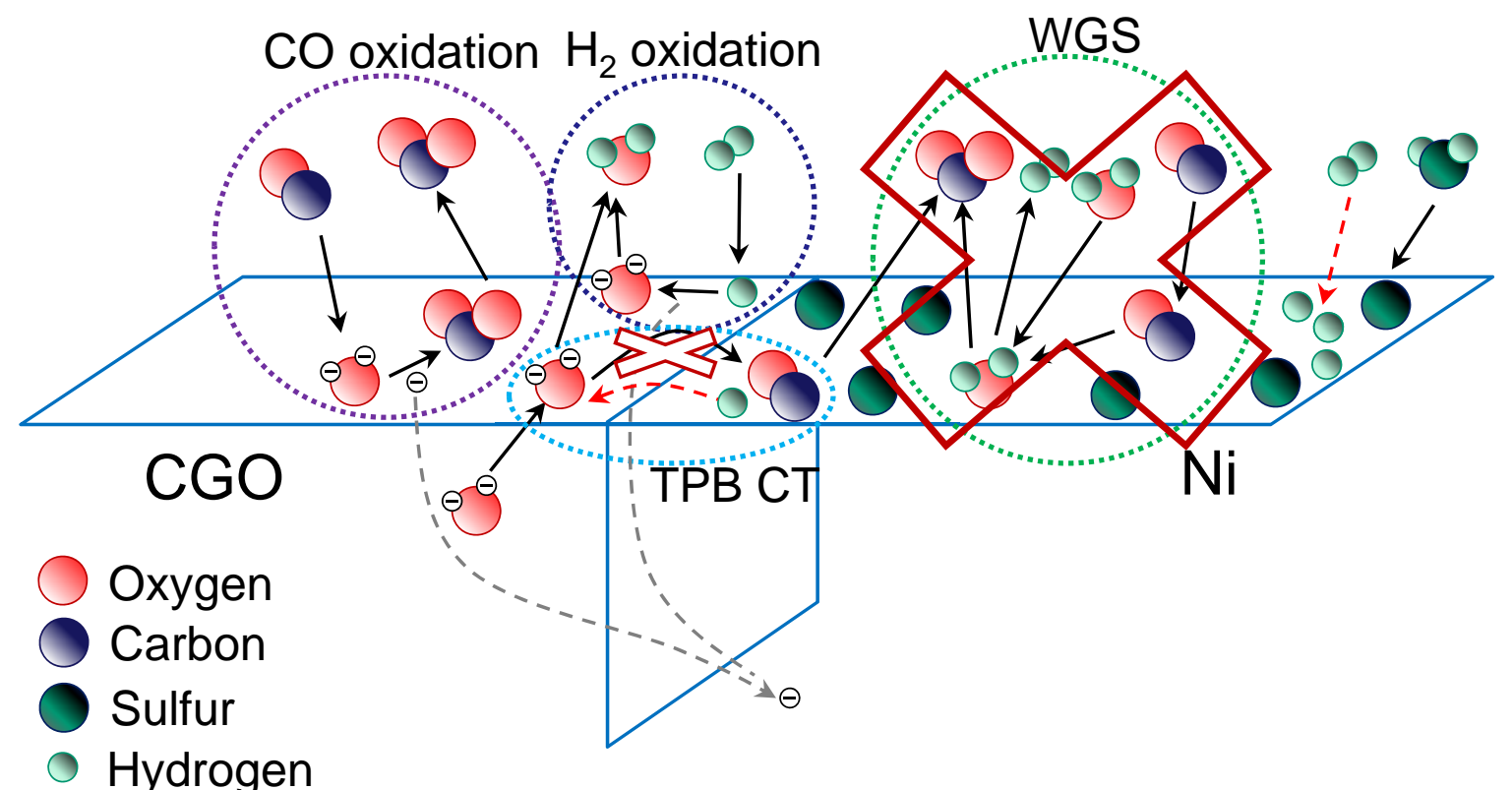

- Ni surface is blocked $\rightarrow \mathrm{CO}$ oxidation at TPB deactivated

- Surface process on CGO is still active (Mars-van-Krevelen mechanism)

- $\mathrm{H}_{2}$ oxidation at TPB probably still active (smaller atom diameter)
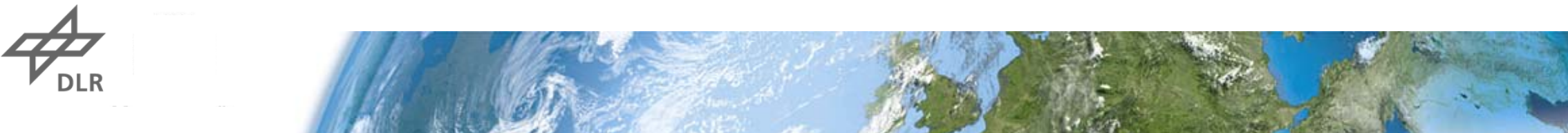


\section{Summary and conclusions}

- Characterization of sulfur poisoning of Ni/CGO anodes in a variety of different reformate fuels

- $\mathrm{Ni} / \mathrm{CGO}$ are able to convert CO even under common sulfur poisoning conditions

- Methan steam reforming on $\mathrm{Ni} / \mathrm{CGO}$ is completely blocked

- The water gas shift reaction is fully deacivated as well

- Electrochemical CO oxidation on CGO is possible 


\section{Acknowledgments}

- Dr. Mihails Kusnezoff and Dr. Nikolai Trofimenko from Fraunhofer IKTS for the supply of cells

- High Temperature Materials Division (HTM) sponsored travel grant

- Financial support from the German Ministry of Education and Research (BMBF) in the framework of the "SOFC Degradation" (Verbundvorhaben SOFC Degradation: Analyse der Ursachen und Entwicklung von Gegenmaßnahmen) project.

Thank you for your attention!
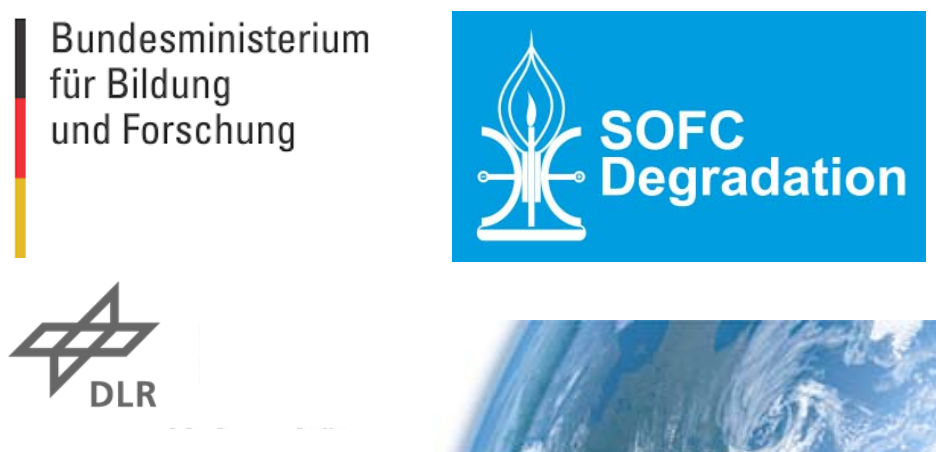